Europe's Journal of Psychology, 8(1), pp. 112-138, doi:10.5964/ejop.v8i1.303

www.ejop.org

\title{
Human Responses to Disasters: A Pilot Study on Peritraumatic Emotional and Cognitive Processing
}

\author{
Anna Grimm, \\ Department of Health and Prevention, Institute of Psychology, University of \\ Greifswald, Germany \\ Lynn Hulse
}

Fire Safety Engineering Group, University of Greenwich, UK

Silke Schmidt

Department of Health and Prevention, Institute of Psychology, University of Greifswald, Germany

\section{Abstract}

This research article presents the qualitative development and cross-cultural pilot testing of a new instrument measuring emotional and cognitive processing during disasters. The instrument was developed according to a theoretical framework based on narratives from survivors of different types of disaster across Europe. Peritraumatic emotions and cognitions were assessed at three different stages of a disaster. The pilot study consisted of 311 participants responding to the questionnaire using scenario versions of disasters as well as 25 survivors working through the questionnaire using their experiences of real disasters. Both types of analysis were performed across seven countries. Differences in emotions and cognitions during the course of a disaster were displayed. Also, gender, the type of scenario participants were allocated to, and professional experience of emergencies led to differences in item response. As there was little difference between survivors' and scenario participants' responses, the use of a scenario in order to test pilot forms of questionnaires for purposive samples with certain characteristics such as limited sizes or access can be supported. For future research, the instrument should be field tested. It is envisaged it will be beneficial for a cross-cultural understanding of the influence of peritraumatic emotions and cognitions not only on posttraumatic

This is an open access article distributed under the terms of the Creative Commons Attribution License (http://creativecommons.org/licenses/by/3.0), which permits unrestricted use, distribution, and reproduction in any medium, provided the original work is properly cited. 
psychological outcomes but also on related behavioural responses displayed during disasters.

Keywords: Cross-cultural development, disaster research, pilot study, peritraumatic emotion and cognition, risk perception

In the last few decades several major disasters have struck Europe, for example: floods in Poland, 2010, Czech Republic and Germany, 2002; earthquakes in L'Aquila, Italy, 2009 and Marmara, Turkey, 1999; plus bombings in London, 2005 and Madrid, 2004. Of interest is whether people's responses to disasters can be generalized or whether they will be unique according to the specific circumstances. That is, will people's behaviour during a disaster follow a similar general pattern or will it vary according to surrounding factors such as the people's prior knowledge and encounters going into the event, what they think and feel as the event unfolds, the environment in which the event occurs and to which the people belong, or even the type of event itself? There is reason to believe these circumstances surrounding people's experiences of disasters may be relevant for their responses to disasters and may even interact with one another. For example, while fires and natural catastrophes such as floods, storms and earthquakes are among the most common disasters in Europe (Preventionweb, n.d.), public and media attention is mainly drawn to man-made disasters such as terror attacks (Grimm, Hulse, \& Schmidt, 2009). Therefore it is possible that people's perception of the threat or significance posed by disasters may vary according to the type of disaster instead of, or in addition to, their exposure to disasters. Regarding people's feelings, fear is often the most reported emotion experienced during disasters (e.g. Prati, Catufi, \& Pietrantoni, 2012; Sotgiu \& Galati, 2007). However, while the specific characteristics of basic emotions like fear, such as rapid onset, automatic appraisal, certain aspects of antecedent events, etc., are universal (Ekman, 1992; Ekman \& Friesen, 1971), recent research suggests that the intensity of emotional responses and cognitive appraisals during disasters differs across cultures. A study examining narratives from European disaster survivors found that not only did the narratives differ significantly across countries in the amount of reported emotional or anxiety words but also in the use of descriptions of cognitive processing (Freitag, Grimm, \& Schmidt, 2011). Also, culture's influence has been shown on risk perception without it being related to rates of actual exposure to terror attacks or tsunamis (Gierlach, Belsher, \& Beutler, 2010) or explained by individual exposure to terror attacks or symptoms of posttraumatic stress disorder (PTSD) (Steger, Frazier, \& Zacchanini, 2008). 
The above research may demonstrate links between several surrounding circumstances, but can one go a step further and draw a link between these factors and people's behavioural responses during disasters? Several recent research studies focusing on survivor narratives have found hints that human behaviour during disasters (e.g. preparing for evacuation, seeking information about the situation, etc.), as well as the victim's surroundings (e.g. being in a familiar place, being with relatives/known persons), can be relevant to not just emotional/cognitive processing during the event but also later posttraumatic stress (Grimm, Hulse, Preiss, \& Schmidt, 2011 a; Prati, Catufi, \& Pietrantoni, 2012; Sotgiu \& Galati, 2007).

People's post-event responses to disasters, such as posttraumatic stress symptoms, have received relatively greater attention from researchers and some of this work has highlighted the influence of certain surrounding circumstances. For example, several theoretical models and frameworks for the explanation of PTSD have a focus on cognitive processing (Brewin \& Holmes, 2003; Ehlers \& Clark, 2000). In their cognitive model of PTSD, Ehlers and Clark (2000) distinguish data-driven processing and conceptual processing during the traumatic event. While conceptual processing is described as an analytic and calm view of the situation, data-driven processing refers to a very sensual and emotional experience mainly in accordance with bewilderment. The latter is said to contribute to PTSD symptoms and disorganized memory functions (Ehlers \& Clark, 2000; Halligan, Michael, Clark, \& Ehlers, 2003). In a meta-analysis, peritraumatic psychological processes were found to be the strongest predictor of PTSD (Ozer, Best, Lipsey, \& Weiss, 2003) and a growing body of research has demonstrated the influence of peritraumatic distress and dissociation on posttraumatic stress outcome (Birmes et al., 2005; Brunet et al., 2001; Fikretoglu et al., 2006; Marmar et al., 1994).

Taken together, the above literature emphasizes the need to consider the specific circumstances surrounding people's experiences of disasters when attempting to understand their responses to disasters - responses both during the event and afterwards. In particular, the research literature suggests directing attention to the circumstances involving how people think and feel during the event. However, in a study where different types of disasters were considered, although peri- and posttraumatic outcome variables were significantly correlated, the event with the highest retrospectively reported peritraumatic emotional stress and perceived risk was not the one with the highest posttraumatic stress (Grimm, Hulse, Preiss, \& Schmidt, $2011 \mathrm{~b}$ ). Thus, there is clearly much still to learn about the influence of specific circumstances on responses to disasters and the nature of the relationships between the circumstances and the responses. The aim of this study was to take a 
first step at addressing this issue by developing a new self-report instrument and conducting a cross-cultural pilot study on emotional and cognitive processing during disasters. It should be noted that a few measures of peritraumatic states already exist, e.g. the Peritraumatic Distress Inventory (Brunet et al., 2001) and the Peritraumatic Dissociative Experience Questionnaire (Marmar et al., 1997). However, despite their good reliability and validity and therefore clear value in many cases, it was felt that they would not be appropriate for the purposes of the present study. It was considered important to (i) have a broader array of emotional and cognitive state options in order to allow investigation of the links with peritraumatic behavioural responses and (ii) compare these states across a number of stages throughout the course of the event as disasters often unfold in a dynamic manner and peritraumatic responses may need to be adaptive. Furthermore, as this study was part of a wider study seeking to improve public safety by examining aspects of physical structures as well as the human behaviour displayed within them, it was preferential to tailor the instrument to disasters involving evacuation attempts from enclosed settings. Finally, as there is reason to believe that culture may impact on human responses to disasters, a simultaneous cross-cultural development of the instrument was conducted.

\section{Aims}

The piloting of the questionnaire had several aims:

- to determine item characteristics

- to assess difficulties and relevance of items in order to identify questions best representing emotional and cognitive processing during disasters

- to assess the influence of certain individual and event characteristics for a better understanding of the constructs (content validation)

- to analyse scale characteristics of anticipated emotional and cognitive responses during disasters plus perceived risk of different types of disasters with explanatory factor analysis (construct validation)

Method

The study described in this paper is part of a larger cross-cultural multi-centre research project called BeSeCu (Behaviour, Security, Culture) with the following centres participating: Greifswald, Germany; London, UK; Barcelona, Spain; Warsaw, Poland; Hamburg, Germany; Prague, Czech Republic; Stockholm, Sweden; Bologna, 
Italy and Izmir, Turkey. The study was approved in all national institutional ethics committees.

Development

The instrument for disaster survivors was developed through two approaches. Firstly, theoretical models and empirical data relating to disasters and peritraumatic factors were reviewed. Secondly, qualitative (single or group) interviews with 125 European survivors of fires, floods, earthquakes and terror attacks were assessed. These actions led to a theoretical framework about emotional, cognitive and behavioural responses during disasters being created (Grimm, Hulse, Preiss, \& Schmidt, 2011 a). Items for the pilot questionnaire were generated from the theoretical framework. Item generation was performed by an expert group consisting of personnel from three countries (Czech Republic, Germany, UK) and took place at an international meeting of the BeSeCu-consortium. The quantity of statements about emotional and cognitive processing in the theoretical framework was considered as an inclusion criteria. The development of the pilot questionnaire was conducted in English. After being finalized, each question was translated and assessed in the national languages of the other participating centres. A manual was designed in order to assist centres during recruitment, the assessment process and data entry, and a data matrix was sent to the centres into which the data could be entered and stored.

Procedure

There are often great challenges in identifying and accessing disaster survivors for research studies, thus it was decided that the pilot study would use scenarios of disasters. This would mean that anyone could take part in the study, regardless of disaster experience and recruitment of real disaster survivors could be mainly reserved for a final field survey. Therefore participants were given a description either of a terror attack, a fire, a flood or an earthquake that was based on the survivor narratives from the aforementioned qualitative interviews (s. Appendix A). However, each centre did additionally recruit a small sample of real survivors to take part in a cognitive debriefing. In this debriefing, the questionnaire was read out loud in the form of a structured interview and the survivors were invited to comment on the questionnaire's structure, understanding and usability. On average, the questionnaire took 45 minutes to be completed and the cognitive debriefing one hour. 
Measurement

The pilot form of the questionnaire consisted of questions about (anticipated) behavioural, emotional and cognitive responses during disasters plus questions about general emergency knowledge, risk perception and socio-demographic data. Considering the scope of this paper, only the analysis of two domains - the peritraumatic factors emotional and cognitive processing, consisting of 14 items, and risk perception, consisting of four items - is presented.

In order to create a more detailed representation of human responses as a disaster unfolds, the questionnaire was separated into three distinct stages of a disaster: realization (of what is happening), during evacuation and after evacuation, and peritraumatic emotions and cognitions were measured at each stage. Risk perception was only assessed once along with general emergency knowledge at the end of the questionnaire. For the pilot study, participants' responses were recorded using a five-point Likert scale, with $0=$ not at all, $1=$ a little, $2=$ moderately, $3=$ strongly and $4=$ very strongly.

Peritraumatic emotion. When taking a look at peritraumatic responses to disasters, emotions play a key role, especially when considering their traits such as quick onset, automatic appraisal and unbidden occurrence (Ekman, 1992). Survivors from the aforementioned interviews did not report a great variety of emotional states, mainly peritraumatic detachment, fear and "panic" (Grimm, Hulse, Preiss, \& Schmidt, 2011 a). Other states reported less often were anger, sadness and depression. States assessed in the Peritraumatic Distress Inventory's negative emotions scale (Brunet et al., 2001), such as guilt and shame or feeling horrified/ helpless were seldom reported by the interviewees and therefore omitted from the piloted instrument. However, an item referring to the concept of controlling one's emotions was assessed in the peritraumatic cognition scale. Fear, one of the strongest predictors for psychological distress following a disaster (Başoğlu, Kiliç, Salcioğlu, \& Livanou, 2004; Başoğlu, Salcioğlu, Livanou, 2002), was assessed using the gradations survivors reported during interviews: nervous, scared, and scared to death. Additionally, the peritraumatic feeling of anger was assessed with the items angry and annoyed. As "panic" during disasters has been reported by survivors in current interview studies, albeit with differing quantities of statements (Grimm, Hulse, Preiss, \& Schmidt, 2011a; Prati, Catufi, \& Pietrantoni, 2012), it was decided to include this item in the questionnaire. Although survivors reported experiencing dissociation and depersonalization during the disaster, it was decided not to integrate dissociative symptoms into the stages part of the questionnaire, for two reasons. Firstly, recent studies support the finding that peritraumatic distress and dissociation are conceptually different, both 
predicting in unique ways PTSD symptoms (Birmes et al., 2005; Fikretoglu et al., 2006). Secondly, dissociation is closely linked with memory disorganization (Halligan et al., 2003). Therefore it was decided that if dissociation were to be of interest, its investigation would be better served by a separate, single assessment using a measure such as the Peritraumatic Dissociative Experience Questionnaire (Marmar et al., 1997).

Leach $(1994 ; 2004)$ has defined three categories of human responses to disasters: calm; reflexive, almost automatic behaviour; and counterproductive reactions. It was decided that the manner of emotional reaction should also be assessed using the items calm and, as its opposite, excited as both were in accordance with Leach's theory and reported by interviewed survivors (Grimm, Hulse, Preiss, \& Schmidt, 2011 a; Leach, 1994; Leach, 2004).

Peritraumatic cognition. Cognitive appraisals of peritraumatic emotions as well as of the disaster situation were assessed with the constructs coping strategies, perceived threat and control beliefs. During disasters, participants' thoughts may centre on comprehending the incident and appraising the situation for risk (Grimm, Hulse, Preiss, \& Schmidt, 2011a). As a traumatic event can also be classified from an individual perspective as an emotional and cognitively overwhelming situation (Ehlers \& Clark, 2000), victims' coping strategies were of interest. As several interviewed survivors reported employing strategies to help deal with the traumatic situation, their statements were reworded in order to generate items. Coping strategies presented in the pilot form were controlling the emotions, focusing on surviving and blocking the situation out. However, it must be noted that such strategies were not reported that often. Feedback indicated that this was either because emotional, cognitive and behavioural responses seemed to happen automatically (s. domain peritraumatic emotion) or because participants could either not remember applying or did not apply any coping strategies.

Leach (1994) suggests that a threat perception is, from a survival-psychological view, an appraisal occurring when people face the possible occurrence of a disaster, which is mainly accompanied, at least initially, by the behavioural components inactivity and denial. In the aforementioned interviews, participants reported the perceived risk of danger to life and health. As the influence of perceived life threat is related not only to greater posttraumatic distress (Hollifield, Hewage, Gunawardena, Kodituwakku, \& Weerarathnege, 2008; Johannesson et al., 2009; Ozer, Best, Lipsey, \& Weiss, 2003; Sumer, Karanci, Berument, \& Gunes, 2005) but also to a possible change in behavioural reactions during disasters (Leach, 1994; Prati, Catufi, \& Pietrantoni, 2012), it was decided that aspects of threat should be assessed in the pilot form. 
Perceived threat was assessed as being concerned about one's safety; this was in order to reflect both the cognitive and the emotional aspects of the construct. A similar item "I felt afraid for my safety" is part of the Peritraumatic Distress Inventory, appearing in the scale on perceived life threat and bodily arousal (Brunet et al., 2001).

Internal locus of control or personal control beliefs are usually assessed as a stable construct, functioning as a resilience factor to posttraumatic distress after disasters (Mellon, Papanikolau, \& Prodromitis, 2009; Sumer, Karanci, Berument, \& Gunes, 2005). However, perceived control over the traumatic situation as it was happening could be related to posttraumatic outcomes as well, as persons with higher PTSD levels might tend to generalize from the state of having no control during the disaster to an overall trait of having no control over their lives (Ehlers \& Clark, 2000). In the pilot study, the assessment of control beliefs was limited to cognitions during the incident; items were created using internal locus of control and external loci of control derived from interviewee narratives.

Risk perception. Risk perception consists of two domains, general/ objective risk and personal/ subjective risk, which are well established in risk research and have been assessed in studies dealing with the influences and consequences of risk perception after terror attacks and natural hazards in affected geographical areas or nationwide (Fischhoff, Gonzalez, Small, \& Lerner, 2003; Goodwin, Wilson, \& Gaines, 2005; Ho et al., 2008; Huddy et al., 2002; Kellens et al., 2011). Huddy et al. (2002) showed that general and personal risk are, although correlated, different dimensions; general risk involves people's opinions about the likelihood/ probability of events occurring in the future in a country or area, while personal risk is connected to emotional and behavioural constructs such as anxiety, fear and somatic symptoms or avoidance behaviour (Goodwin, Wilson, \& Gaines, 2005; Huddy et al., 2002). Therefore it was believed that the assessment of personal risk would be possible with the use of disaster scenarios and could reveal something of interest regarding answering tendencies about anticipated cognitive and emotional responses to disasters. It was expected that the construct validity of the questionnaire would be enhanced if personal risk loaded on factors other than just peritraumatic emotional and cognitive responses to a hypothetical disaster scenario. Following the approach taken in other studies, it was decided that personal risk would be assessed as concern (Goodwin, Wilson, \& Gaines, 2005; Huddy et al., 2002; Kellens et al., 2011). 
Participants

Recruitment was performed by each centre in November 2009. Scenario participants were required to be aged between 18 and 60 years. For the cognitive debriefings, participants had to have already experienced one of the following disasters: a flood, a fire, an earthquake, or a terror attack. After giving consent, participants completed a paper/ pencil version of the pilot questionnaire. Questionnaire assessment and cognitive debriefings were conducted using experienced psychologists.

A total of 336 participants took part in the study; 53 from Germany, 20 from the UK, 53 from Spain, 50 from Sweden, 52 from Poland, 54 from the Czech Republic, 48 from Turkey and six from Italy. There was an almost even split of females (55.1\%) and males (44.9\%). Mean age was 35.5 years (SD $=13.5$ ). Three percent of participants had a migrant background; as this was a small percentage, migrant status was omitted from the analysis. On average, participants had been in education for 16.0 years $(S D=5.9)$. Twenty percent of participants had experience of working as a police officer or firefighter. Twenty-five participants had already survived a fire, a flood or an earthquake. These events happened on average ten years (SD= 8.5) ago. Participants were divided into the scenarios fire (40\%), terror attack (20\%), earthquake $(20 \%)$ and flood $(17 \%)$. The remaining three percent took part in the cognitive debriefings.

Statistical Analysis

Questionnaire responses were coded and data checked in a standardized PASW/ Excel data matrix by each participating centre. After this, the data matrix and completed questionnaires were sent to the coordinating centre in Greifswald for final checking and data analysis. Implausible data or values out of range were coded as missing if they could not be verified in the completed questionnaire. If two answers were coded instead of a single answer, two raters decided which one to pick. All statistical analyses were conducted with PASW version 18.0. Several tests were applied in order to analyse item and scale characteristics. Descriptive statistics including means and standard deviations were calculated. Effects of gender, scenario type, disaster experience and emergency work experience were analysed with T-tests and univariate ANOVAs. Differences between the three disaster stages were assessed with repeated measures ANOVAs. Scale characteristics were analysed with Cronbach's $\alpha$, item-scale correlations and scale intercorrelations were assessed with bivariate Pearson-correlations. The structure of the pilot form was assessed with explanatory factor analysis. Regarding item and scale characteristics, 
an item was deleted if its substance was diverged, but also if the item-scale correlation was below .30 at all three disaster stages and the scale's Cronbach's $\alpha$ was enhanced by deleting the item.

Results

\section{Cognitive debriefing}

In general, the questionnaire sections on peritraumatic emotions and cognitions were considered a good fit to the participants' disaster experiences. Also the structure of the questionnaire, with the repetition of the set of questions about peritraumatic emotions and cognitions at three disaster stages, was found useful in order to illustrate a dynamic experience of peritraumatic states. Feedback included suggestions to simplify or reword several items, especially on the coping strategies and control beliefs scales, in order to increase ease of understanding, e.g. remove double negatives in one sentence. Also several participants found that items about coping strategies were not always applicable in their given situation. Questions rated as unsuitable during the cognitive debriefings were verified again by researchers but only omitted if unsuitability was also indicated by psychometric criteria. A further point raised was that different gradations of fear, such as the items scared and scared to death, were overly similar. Thus, as a consequence of participant feedback and psychometric analysis, items were removed.

Item characteristics

In a first step, three items, one from the emotion scale (calm) and two from the control beliefs scale (control self/ others) were deleted due to low item-scale correlations and changes in Cronbach's $\alpha$. One further item, being excited, was translated in some countries with a negative connotation and in others with a positive connotation, and was therefore removed by expert consent. Gender differences were apparent on $10-30 \%$ of items (depending on the stage of the disaster), predominantly on the emotion scale. For about $60 \%$ of all items, the type of scenario participants were allocated to resulted in response differences at the first two disaster stages; differences decreased at the after evacuation stage. Scenario differences were mainly apparent on the emotion and risk scales. When comparing the realization stage items to the during evacuation and after evacuation stage items, significant differences were found on all items except for the blocking the situation out item. Mean item scores decreased significantly across the three stages, except for the being angry item, on which the mean scores increased. Results for the characteristics of the remaining items are shown in Tables 1, 2 and 3. 
Table 1: Characteristics (after the scale revisions) of items on peritraumatic emotion at the different disasters stages ( $\mathrm{n}=336$ )

\begin{tabular}{|c|c|c|c|c|c|c|c|c|c|c|c|c|}
\hline \multirow{2}{*}{$\begin{array}{l}\text { Scale: } \\
\text { Peritraumatic Emotion }\end{array}$} & \multicolumn{4}{|c|}{ Stage 1): Realization } & \multicolumn{4}{|c|}{ Stage2): During Evacuation } & \multicolumn{4}{|c|}{ Stage 3): After Evacuation } \\
\hline & $M(S D)$ & Gen & Sce & ISC & $M(S D)$ & Gen & Sce & ISC & $M(S D)$ & Gen & Sce & ISC \\
\hline I was annoyed. * & $1.38(1.31)$ & & $\bullet \bullet$ & .52 & $1.20(1.22)$ & & $\bullet \bullet$ & .60 & $1.25(1.28)$ & & & .70 \\
\hline I was angry. ${ }^{* *}$ & $0.91(1.19)$ & & & .60 & $0.83(1.11)$ & & & .64 & $1.09(1.30)$ & & & .71 \\
\hline I was nervous. *** & $2.15(1.34)$ & $\bullet \bullet$ & $\bullet \bullet \bullet$ & .74 & $1.93(1.24)$ & & $\bullet \bullet \bullet$ & .72 & $1.60(1.19)$ & $\bullet$ & & .65 \\
\hline I was scared. *** & $2.37(1.25)$ & $\bullet \bullet \bullet$ & $\bullet \bullet$ & .80 & $1.91(1.30)$ & $\bullet \bullet \bullet$ & $\bullet \bullet \bullet$ & .83 & $1.28(1.19)$ & $\bullet \bullet$ & $\bullet \bullet$ & .78 \\
\hline I was panicking. *** & $1.29(1.33)$ & $\bullet \bullet$ & $\bullet \bullet \bullet$ & .79 & $1.00(1.26)$ & $\bullet \bullet$ & $\bullet \bullet \bullet$ & .84 & $0.49(0.91)$ & & $\bullet \bullet \bullet$ & .70 \\
\hline I was scared to death. ${ }^{* * *}$ & $1.17(1.38)$ & $\bullet \bullet$ & $\bullet 00$ & .79 & $1.02(1.37)$ & & $\bullet \bullet \bullet$ & .77 & $0.48(0.99)$ & & $\bullet \bullet \bullet$ & .66 \\
\hline
\end{tabular}

Note: Gen = Gender, Sce = Scenario, ISC = Item Scale Correlation $(\bullet=p<.05 ; \bullet \bullet=p<.01 ; \bullet \bullet \bullet=p<.001)$

Within subject differences between stages: ${ }^{*}=p<.05 ;{ }^{* *}=p<.01 ;{ }^{* * *}=p<.001$ 
Table 2: Characteristics (after the scale revisions) of items on peritraumatic cognitions at the different disaster stages ( $\mathrm{n}=336$ )

\begin{tabular}{|c|c|c|c|c|c|c|c|c|c|c|c|c|}
\hline \multirow{2}{*}{$\begin{array}{l}\text { Scale: } \\
\text { Peritraumatic Cognition }\end{array}$} & \multicolumn{4}{|c|}{ Stage 1): Realization } & \multicolumn{4}{|c|}{ Stage2): During Evacuation } & \multicolumn{4}{|c|}{ Stage 3): After Evacuation } \\
\hline & $M(S D)$ & Gen & Sce & ISC & $M(S D)$ & Gen & Sce & ISC & $M(S D)$ & Gen & Sce & ISC \\
\hline $\begin{array}{l}\text { How concerned were you } \\
\text { about your safety? }{ }^{* * *}\end{array}$ & $2.54(1.08)$ & $\bullet \bullet$ & $\bullet \bullet$ & .63 & $2.20(1.12)$ & $\bullet \bullet$ & $\bullet \bullet$ & .72 & $1.33(1.16)$ & & & .55 \\
\hline $\begin{array}{l}\text { The situation was out of } \\
\text { control. *** }\end{array}$ & $1.43(1.31)$ & & $\bullet \bullet \bullet$ & .69 & $.92(1.12)$ & & $\bullet \bullet \bullet$ & .64 & $.49(.82)$ & & $\bullet \bullet \bullet$ & .46 \\
\hline $\begin{array}{l}\text { The situation was in the } \\
\text { hands of fate. }{ }^{* * *}\end{array}$ & $1.16(1.35)$ & & $\bullet \bullet$ & .57 & $.75(1.11)$ & & $\bullet \bullet$ & .57 & $.57(1.01)$ & $\bullet$ & $\bullet \bullet \bullet$ & .47 \\
\hline $\begin{array}{l}\text { I tried to control my } \\
\text { feelings. ** }\end{array}$ & $2.32(1.08)$ & & & .33 & $2.26(1.04)$ & & & .29 & $2.07(1.12)$ & & & .69 \\
\hline I blocked the situation out. & $1.25(1.25)$ & & & .39 & $1.25(1.22)$ & $\bullet$ & & .53 & $1.46(1.27)$ & & & .54 \\
\hline I focused on surviving. ${ }^{* * *}$ & $2.61(1.12)$ & & & .59 & $2.29(1.26)$ & & & .69 & $1.56(1.30)$ & & & .60 \\
\hline
\end{tabular}

Note: Gen = Gender, Sce = Scenario, ISC = Item Scale Correlation $(\bullet=p<.05 ; \bullet \bullet=p<.01 ; \bullet \bullet \bullet=p<.001)$

Within subject differences between stages: ${ }^{*}=p<.05 ; * *=p<.01 ; * * *=p<.001$ 
Table 3: Characteristics (after the scale revisions) of items on personal risk $(n=336)$

\begin{tabular}{|c|c|c|c|c|}
\hline Personal risk & $M(S D)$ & Gen & Sce & ISC \\
\hline $\begin{array}{l}\text { How concerned are you about becoming the } \\
\text { victim of a fire? }\end{array}$ & $1.36(0.97)$ & & $\bullet \bullet$ & .74 \\
\hline $\begin{array}{l}\text { How concerned are you about becoming the } \\
\text { victim of a terror attack? }\end{array}$ & $1.11(1.13)$ & & $\bullet \bullet \bullet$ & .85 \\
\hline $\begin{array}{l}\text { How concerned are you about becoming the } \\
\text { victim of a flood? }\end{array}$ & $1.08(1.11)$ & & $\bullet \bullet \bullet$ & .75 \\
\hline $\begin{array}{l}\text { How concerned are you about becoming the } \\
\text { victim of an earthquake? }\end{array}$ & $0.86(1.22)$ & & $\bullet \bullet \bullet$ & .84 \\
\hline
\end{tabular}

Note: Gen $=$ Gender, Sce $=$ Scenario, ISC = Item Scale Correlation $(\bullet=p<.05$;

$\bullet \bullet=p<.01 ; \bullet \bullet \bullet=p<.001)$

Influence of experience

Few differences in item responses were found between the disaster survivors who completed a cognitive debriefing and scenario participants. However, survivors did rate the situation as being out of control at a significantly higher level for the realization and after evacuation stages $(T=-2.08 ; \mathrm{p}<.05$ and $\mathrm{T}=-2.36 ; \mathrm{p}<.05$, respectively), while scenario participants gave a significantly higher endorsement of the item about blocking the situation out at all disaster stages ( $T=2.08 ; \mathrm{p}<.05$, $\mathrm{T}=2.93 ; \mathrm{p}<.01$ and $\mathrm{T}=2.78 ; \mathrm{p}<.05$, respectively). Moreover, the item about controlling one's feelings was rated higher by scenario participants at the during evacuation stage $(T=2.28 ; \mathrm{p}<.05)$.

Differences between participants who had experience of working for the emergency services ("emergency workers") and participants with no emergency work experience ("civilians") were found particularly at the realization and during evacuation stages. The items about being nervous $(T=2.99 ; \mathrm{p}<.01$ and $\mathrm{T}=2.33$; $\mathrm{p}<.05$, respectively) and the situation being out of control $(\mathrm{T}=2.80 ; \mathrm{p}<.01$ and $\mathrm{T}=$ 3.09; $p<.01$, respectively) differed significantly at both stages, with civilians scoring higher than emergency workers. Also, at the realization stage, civilians reported higher levels of concern about their own safety $(\mathrm{T}=3.39 ; \mathrm{p}<.01)$ and being scared to death ( $\mathrm{T}=3.00 ; \mathrm{p}<.01)$, while emergency workers reported significantly higher levels of being annoyed $(T=2.84 ; \mathrm{p}<.01)$. At the third disaster 
stage there were no differences between emergency workers and civilians. However, emergency workers were more concerned about becoming a victim of a fire $(T=-3.07 ; p<.01)$ and an earthquake than were civilians $(T=-2.59 ; \mathrm{p}<.05)$.

\section{Scale characteristics}

The principal component analysis (using Varimax) explained altogether $66.6 \%$, $62.3 \%$ and $65.3 \%$ of variance for the three disaster stages, respectively. The first factor included four items about the emotional states of fear and panic; at the realization and during evacuation stages, the items about concern and focusing on surviving were also included. The second factor included four items about personal risk at all stages. The third factor had two items regarding anger; at the after evacuation stage this factor also included feeling nervous. Finally, the fourth factor included the coping strategies items and the fifth factor the two remaining control beliefs items. Cronbach's $\alpha$ for the peritraumatic emotion, cognition, and the personal risk scales are shown in Table 4 before and after revisions.

Table 4: Scale characteristics: Cronbach's $\alpha$ before and after revisions

\begin{tabular}{|c|c|c|c|c|c|}
\hline \multirow{2}{*}{$\begin{array}{l}\text { Scale } \\
\text { Revision }\end{array}$} & \multicolumn{2}{|c|}{$\begin{array}{l}\text { Peritraumatic } \\
\text { Emotion }\end{array}$} & \multicolumn{2}{|c|}{$\begin{array}{l}\text { Peritraumatic } \\
\text { Cognition }\end{array}$} & \multirow[t]{2}{*}{ Personal Risk } \\
\hline & Before & After & Before & After & \\
\hline $\begin{array}{l}\text { Stage 1): } \\
\text { Realization }\end{array}$ & .66 & .83 & .57 & .50 & \\
\hline $\begin{array}{l}\text { Stage 2): During } \\
\text { Evacuation }\end{array}$ & .70 & .84 & .45 & .60 & \\
\hline $\begin{array}{l}\text { Stage 3): After } \\
\text { Evacuation }\end{array}$ & .65 & .78 & .32 & .54 & .82 \\
\hline
\end{tabular}

The peritraumatic emotion and cognition and the personal risk scales were all significantly intercorrelated at all disaster stages. Scale intercorrelations are shown in Table 5. Correlations were beneficial in showing that all scales belonged 
to a superordinate construct, but the correlations were not high enough to conclude that the scales were measuring the same thing.

Table 5: Scale intercorrelations (after the revisions) for peritraumatic emotions and cognitions and personal risk

\begin{tabular}{|c|c|c|c|}
\hline Stage & Scale & $\begin{array}{l}\text { Scale II: Peritraumatic } \\
\text { Cognition }\end{array}$ & $\begin{array}{l}\text { Scale III: } \\
\text { Personal Risk }\end{array}$ \\
\hline Stage 1) & Scale I: Peritraumatic & $.54^{* * *}$ & $.15^{*}$ \\
\hline \multirow[t]{3}{*}{ Realization } & Emotion & & \\
\hline & Scale II: Peritraumatic & - & $.27^{* * *}$ \\
\hline & Cognition & & \\
\hline Stage 2) & Scale I: Peritraumatic & $.56^{* * *}$ & $.19^{* *}$ \\
\hline During & Emotion & & \\
\hline Evacuation & Scale II: Peritraumatic & - & $.36^{* * *}$ \\
\hline & Cognition & & \\
\hline Stage 3) & Scale I: Peritraumatic & $.34^{* * *}$ & $.23^{* * *}$ \\
\hline After & Emotion & & \\
\hline \multirow[t]{2}{*}{ Evacuation } & Scale II: Peritraumatic & - & $.25^{* * *}$ \\
\hline & Cognition & & \\
\hline
\end{tabular}

$*=p<.05 ; * *=p<.01 ; * * *=p<.001$

\section{Discussion}

In this study, part of a larger international study of human responses to disasters, a new instrument measuring emotional and cognitive processing during disasters was pilot tested across several European countries. The development of the instrument was based on theoretical models from the fields of trauma-, survival-, and social psychology and a theoretical framework generated from survivor narratives. The process consisted of several steps, from interviews with disaster survivors to item development, design of a pilot questionnaire, plus psychometric and content analysis. Pilot testing was performed according to the approach taken by other international research projects and was considered to be a beneficial way of testing psychometric characteristics and usability in order to enhance the questionnaire (Petersen, Schmidt, Power, \& Bullinger, 2005; Winkler, Matschinger, \& Angermayer, 2006). It was found that, overall, the pilot study 
produced a satisfactory instrument for assessing the constructs peritraumatic emotions, peritraumatic cognitions including perceived threat, coping strategies and control beliefs, as well as personal risk. In general, the pilot study supported the use of a questionnaire teamed with event scenarios, especially when recruitment of a purposive sample - in this case, survivors of disasters - is challenged by factors such as limited access and small sizes. While the usability and feasibility of the questionnaire appeared to be enhanced after taking on board the results from both sets of participants, it must be noted that an empirical validation of the employed models and theoretical framework was not possible as most participants were answering based on a scenario and not personal experience.

When comparing the developed instrument with current standardized measures such as the Peritraumatic Distress Inventory (PDI) (Brunet, et al., 2001), differences as well as similarities can be found. The PDI measures reactions to traumatic events with two scales, negative emotions (7 items) and perceived life threat/ bodily arousal (6 items). Both measures contain similar items regarding emotions. In the PDI, the concept of anger is represented as anger/ frustration about not being able to do more, while the developed instrument measures being angry/ annoyed in general. In addition, the PDI emphasizes the feelings of fear, helplessness and horror during the event, in accordance with the DSM-IV PTSD Criterion A2, while the developed instrument focuses on fear and panic. The items sadness/ grief from the PDI were not assessed in the new instrument as these secondary emotions were mainly reported by interviewed survivors as being experienced after the disaster, not during it. Similarly, the PDI items on guilt and shame were not assessed in the new instrument as these were not reported in survivor narratives. It is possible that such items might be more relevant to traumatic events other than major disasters, e.g. interpersonal traumas such as assaults.

Some differences in the conceptual frameworks of both measures are apparent. An item on controlling emotions was present in both questionnaires; in the PDI it belonged to the negative emotion scale, in the new instrument it was seen as a coping strategy and therefore considered a peritraumatic cognition. Conversely, the item "I thought I might die" came under the PDI's perceived life threat scale, while a similar item "I was scared to death" came under the peritraumatic emotion scale in the new instrument. Furthermore, although perceived life threat was part of both instruments, bodily arousal was not measured in the new one. Fikretoglu et al. (2007) found the effect of peritraumatic negative emotions on 
dissociation was reduced in police officers, and no longer significant for civilians, when peritraumatic physical and cognitive panic reactions were controlled for. Those authors used the PDI in order to asses panic but suggested that future research should instead control for the presence of a panic attack during the traumatic event. Therefore, in the current study, all symptoms of a panic attack were assessed using the DSM-IV criteria. However, due to the scope of this article, the results of that assessment are not presented here. Several new items were created for coping strategies such as focusing on surviving/ blocking the situation out and external loci of control during the disaster. Like all other items, these items were derived from disaster survivors' narratives, however recent research has also highlighted a need to investigate cognitive strategies during traumatic situations such as self-regulation of emotions and focusing on the reality of the threat in order to better understand mechanisms of peritraumatic responses (Briere, Scott, \& Weathers, 2005; Fikretoglu et al., 2006).

In summary, the two measures are similar in some respects but are ultimately different, and not just in the choice of items. The PDI aims to assess if several peritraumatic states were present during a traumatic event and how these influence related states such as peritraumatic dissociation and acute stress disorder (ASD)/ PTSD symptoms. The new instrument was developed to investigate peritraumatic states as the event unfolded, and so incorporated a repeated measures aspect, and the events of interest were specifically disasters, ones which necessitated an evacuation attempt from an enclosed setting. Another aim of the new instrument was to allow the investigation of interactions between peritraumatic emotional and cognitive processing and behavioural responses during a disaster experience.

Effects of gender were detected when considering higher peritraumatic fear/ concern during disasters. Gender effects have also been detected in epidemiological studies on postraumatic stress reactions after the World Trade Center terror attacks (Silver et al., 2002; Schlenger et al., 2002). However, regarding risk perception, no gender effects were detected, which is not in accordance with recent findings (Armaş, 2006; Huddy et al., 2002). The type of scenario did have significant effects on the answering tendencies. Here, it must be noted that all scenarios described a situation of similar risk, from the stage of realization, to the stage where an evacuation was being initiated, through to the stage of a participant reaching a place of complete safety. Regarding differences in peritraumatic fear and cognition related to the type of scenario, it should be noted that other recent research studies have found significant 
differences in survivors' post- and peritraumatic stress depending on the type of disaster they had experienced (Grimm, Hulse, Preiss, \& Schmidt, 2011b; Shakespeare-Finch \& Armstrong, 2010). One possible explanation regarding the scenario effect on personal risk might be that participants were primed; that is, if a participant was given a scenario describing a terror attack for instance, the scenario itself may have increased salience of terror attacks in the participant's mind, which might have then resulted in higher levels of concern of becoming a victim of a terror attack. In future research, the relationship between the type of disaster with its unique characteristics and levels of peri- and posttraumatic stress should be investigated.

The relevance of the stages approach, i.e. assessing cognitions and emotions during different parts of the disaster, was underlined by significant changes in answering tendencies at realization, during evacuation and after evacuation. The authors believe that a dynamic view of peritraumatic responses will help to further investigate adaptive behaviour during disasters but also its influence on posttraumatic stress outcome.

Overall, item responses did not differ much between real survivors and scenario participants. This underlines the quality of the content validity of the questionnaire, which was undoubtedly enhanced by drawing from detailed survivor narratives. However, prior disaster experience did impact on subjective risk concerning one particular disaster type, fire, although statistical tests revealed a trend just short of being significant. The effect of prior disaster experience on future perceived risk has been shown in several studies for floods, landslides and terror attacks (Kellens et al., 2011; Ho et al., 2008; Fischhoff, Gonzalez, Small, \& Lerner, 2003), therefore it is possible that the small number of real disaster survivors in the current sample, and the time that had passed since the incidents (10 years on average), might have produced this non-significant result (see also Helweg-Larsen, 1999). Experience of a different kind, professional emergency experience, had a significant effect on certain answers. Differences between civilians and emergency workers were found at the realization and during evacuation stages on relevant items such as being scared to death, being nervous, being concerned about one's safety and thinking that the situation was out of control, which is in accordance with other studies (Brunet et al., 2001; Fikretoglu et al., 2007).

Regarding the scale structure, it was demonstrated that items were grouped along the factors of emotional and cognitive processing during disasters and 
personal risk. However, it must be noted that, especially for the stages during the disaster, a strict isolation of peritraumatic emotions and cognitions might not be possible, which might be related to the characteristics of basic emotions (Ekman, 1992). The scenario group's answers about peritraumatic emotions and cognitions might have been confounded with the actual concern those people had about becoming a victim of a disaster and, to a lesser extent, related to the disaster scenario itself. Relationships between the peritraumatic emotions/ cognitions and personal risk scales were detected via significant positive correlations. However, although the construct of threat during the event was, considering the wording of the items, somewhat connected to the construct of personal risk, the items did load on different factors. Thus, this finding supports the idea that the scales measure different constructs.

\section{Limitations}

Although scenario assessment worked in achieving improved usability and feasibility of the instrument, it was not without its challenges. For example, this approach relied on participants being able to put themselves 'in the shoes' of a real disaster survivor and being able to embellish on the information given in the scenario description. Answers about emotional and cognitive disaster experiences would have been drawn from layperson beliefs. As Alexander (2007) showed, there are still many misconceptions about human responses to disasters, such as the frequency of occurrence of mass panic, so the results of the pilot form based on scenario participants may have been influenced by underlying misleading constructs. However, this was addressed by undertaking cognitive debriefings with real survivors and taking their results into account along with those of the scenario participants together with psychometric analysis. In addition, it must be considered that, in order to have a realistic illustration of disasters, the scenarios were based on previous interviews with 125 disaster survivors. Furthermore, the same researchers were engaged in those interviews and the pilot development of the new instrument.

It must be noted that neither the number of cognitive debriefings per centre nor the number of interviews per disaster type and country that the final scales were based on should be considered representative and were rather affected by the incidence rates of disasters across Europe. Therefore a systematic bias in item response due to the disasters experienced across countries might have occurred. Also, a self-selection bias might have been present; survivors with disorganized and incomplete memories or heightened emotional states due to severe 
traumatic stress might have avoided taking part in the interviews and cognitive debriefings and so the results on emotions and cognitions might only pertain to survivors with less of a posttraumatic stress outcome (Grimm, Hulse, Preiss, \& Schmidt, 2011 a). As discussed earlier, there were very few significant differences in answers between real survivors and scenario participants and this could also be a function of the low number of real disaster survivors in the sample. A further limitation concerning the validation of the instrument is that current postraumatic stress symptoms (deriving from any specific traumatic life event) were not assessed in the sample. According to Ehlers and Clark's (2000) cognitive model of PTSD, individuals with higher current levels of PTSD use several dysfunctional behavioural and cognitive strategies, which assist in maintaining PTSD symptoms. Therefore it could be assumed that if any of the scenario participants had current PTSD symptoms then they may have tended to anticipate their responses to a disaster scenario to be more passive and stressed. However, while this validation of the instrument is of course necessary it will be more reliably assessed in a study where disaster exposure is controlled for.

Finally, the items on emotional and cognitive states were designed for the disaster types fire, flood, earthquake and terror attack, and where an evacuation was attempted from an enclosed setting; generalizing to other disasters cannot be supported at this point. Although development and assessment was performed in seven European countries, cross-cultural validity is not a given yet. Another aim for future research can be to empirically identify differences in emotions and cognitions (Freitag, Grimm, \& Schmidt, 2011) as well as in perceived risk of disasters across Europe and compare these to differences indicated in literature (Gierlach, Belsher, \& Beutler, 2010; Shiloh, Guvenc, \& Onkal, 2007; Steger, Frazier, \& Zacchanini, 2008).

\section{Conclusion}

In summary, this article presented the cross-cultural development and pilot testing of a new instrument measuring emotions and cognitions during disasters as well as personal risk. The pilot testing was based on psychometric criteria including participants' responses to different types of disaster scenarios and cognitive debriefings involving real disaster survivors. The procedure enhanced the constructs and gave information as to how items could be reformulated for better understanding and usability. Also, pitfalls in cross-cultural research, such as translation problems concerning the connotation of emotion words, were brought to light. Overall, $20 \%$ of the initial items were reduced and three scales - 
peritraumatic emotion, with its facets fear and anger, as well as peritraumatic cognition and personal risk - resulted from the pilot study. Also, the findings highlighted the relevance of investigating emotions and cognitions during different stages of a disaster. Future research should investigate further the influence of peritraumatic emotions and cognitions during the distinct stages of disasters as this will likely be beneficial for the understanding of posttraumatic psychological outcomes, as well as for the understanding of behavioural responses displayed during disasters. Also beneficial would be further studies on cross-cultural differences in the displayed intensity of such emotions and cognitions as fear, anger, perceived threat, coping strategies and individual control beliefs.

\section{Acknowledgements}

The paper was written by Anna Grimm, Lynn Hulse and Silke Schmidt on behalf of the BeSeCu-group. The project BeSeCu (contract 218324) is funded under the European Union Framework 7 Security initiative. The authors acknowledge the co-operation of their project partners: Ernst-Moritz-Arndt University Greifswald, Department of Health and Prevention, Germany (project co-ordinator); University of Greenwich, FSEG, UK; Institute of Public Security of Catalunya, Spain; Hamburg Fire and Emergency Service Academy, Germany; MTO Psykologi, Sweden; Prague Psychiatric Centre, Czech Republic; Main School of Fire Service, Poland; Hamburg Fire and Emergency Service Academy, Germany and Association of Emergency Ambulance Physicians, Turkey; University of Bologna, Italy; in undertaking this work and in allowing the project findings to be published.

\section{References}

Alexander, D. E. (2007). Misconception as a barrier to teaching about disasters. Prehospital and Disaster Medicine, 22(2), 95-103.

Armaş, I. (2006). Earthquake risk perception in Bucharest, Romania. Risk Analysis, 26(5), 1223-1234. doi:10.11 11 /j.1539-6924.2006.00810.x

Başoğlu, M., Salcioğlu, E., \& Livanou, M. (2002). Traumatic stress response in earthquake survivors in Turkey. Journal of Traumatic Stress, 15(4), 269-276. doi:10.1023/A:1016241826589 
Başoğlu, M., Kiliç, C., Salcioğlu, E., \& Livanou, M. (2004). Prevalence of posttraumatic stress disorder and comorbid depression in earthquake survivors in Turkey: An epidemiological study. Journal of Traumatic Stress, 17(2), 133-141. doi:10.1023/B:JOTS.0000022619.31615.e8

Birmes, P. J., Brunet, A., Coppin-Calmes, D., Arbus, C., Coppin, D., Charlet, J.-P., Vinnemann, N., Juchet, H., Lavque, D., \& Schmitt, L. (2005). Symptoms of Peritraumatic and Acute Traumatic Stress among Victims of an Industrial Disaster. Psychiatric Services (Washington, D.C.), 56(1), 93-95. doi:10.1176/appi.ps.56.1.93

Brewin, C. R., \& Holmes, E. A. (2003). Psychological theories of posttraumatic stress disorder. Clinical Psychology Review , 23, 339-376. doi:10.1016/S02727358(03)00033-3

Briere, J., Scott, C., \& Weathers, F. (2005). Peritraumatic and Persistent Dissociation in the Presumed Etiology of PTSD. The American Journal of Psychiatry, 162(12), 2295-2301. doi:10.1176/appi.ajp.162.12.2295

Brunet, A., Weiss, D. S., Metzler, T. J., Best, S. R., Neylan, T. C., Rogers, C., Fagan, J., \& Marmar, C. R. (2001). The peritraumatic distress inventory: A proposed measure of PTSD Criterion A2. The American Journal of Psychiatry, 158, 1480-1485. doi:10.1176/appi.ajp.158.9.1480

Ekman, P. (1992). An argument for basic emotions. Cognition and Emotion, 6, 169-200. doi:10.1080/02699939208411068

Ehlers, A., \& Clark, D. M. (2000). A cognitive model of posttraumatic stress disorder. Behaviour Research and Therapy, 38, 319-345. doi:10.1016/S00057967(99)00123-0

Ekman, P., \& Friesen, W. V. (1971). Constants across cultures in the face and emotion. Journal of Personality and Social Psychology, 17(2), 124-129. doi:10.1037/h0030377

Fikretoglu, D., Brunet, A., Best, S., Metzler, T., Delucchi, K., Weiss, D. S., .Fagan, J. \& Marmar, C. (2006). The relationship between Peritraumatic Distress and Peritraumatic Dissociation. The Journal of Nervous and Mental Disease, 194(11), 853-858. doi:10.1097/01.nmd.0000244563.22864.f1

Fikretoglu, D., Brunet, A., Best, S. R., Metzler, T. J., Delucchi, K., Weiss, D. S., Fagan, J., \& Marmar, C. R. (2007). Peritraumatic fear, helplessness and horror and 
peritraumatic dissociation: Do physical and cognitive symptoms of panic mediate the relationship between the two? Behaviour Research and Therapy, 45, 39-47. doi:10.1016/j.brat.2006.01.008

Fischhoff, B., Gonzalez, R. M., Small, D. A., \& Lerner, J. S. (2003). Judged terror risk and proximity to the World Trade Center. Journal of Risk and Uncertainty, 26(2/3), 137-151. doi:10.1023/A:1024163023174

Freitag, S., Grimm, A., \& Schmidt, S. (2011). Talking about traumatic events: A cross-cultural investigation. Europe's Journal of Psychology, 1, 40-61.

Gierlach, E., Belsher, B. E., \& Beutler, L. E. (2010). Cross-cultural differences in risk perceptions of disasters. Risk Analysis, 30(10), 1539-1549. doi:10.1111/j.1539$6924.2010 .01451 . x$

Goodwin, R., Wilson, M., \& Gaines, S., Jr. (2005). Terror perception and its consequences in contemporary Britain. British Journal of Psychology, 96, 389-406. doi:10.1348/000712605X62786

Grimm, A., Hulse, L., \& Schmidt, S. (2009). Risk perception and psychological reactions in public crisis situations using the example of terror attacks. Bundesgesundheitsblatt, 52(12), 1129-1140. doi:10.1007/s00103-009-0966-6 Grimm, A., Hulse, L., Preiss, M. \& Schmidt, S. (201 1a). Behavioural, cognitive and emotional responses in European disasters: Results of survivor interviews. Manuscript submitted for publication.

Grimm, A., Hulse, L., Preiss, M. \& Schmidt, S. (201 1b). Post- and peritraumatic stress in European disaster survivors: An explorative study about the influence of individual and event characteristics across different types of disasters. Manuscript submitted for publication.

Halligan, S. L., Michael, T., Clark, D. M., \& Ehlers, A. (2003). Posttraumatic stress disorder following assault: The role of cognitive processing, trauma memory, and appraisals. Journal of Consulting and Clinical Psychology, 71 (3), 419-431. doi:10.1037/0022-006X.71.3.419

Helweg-Larsen, M. (1999). (The lack of) optimistic biases in response to the 1994 Northridge earthquake: The role of personal experience. Basic and Applied Social Psychology, 21 (2), 119-129. 
Huddy, L., Feldmann, S., Capelos, T., \& Provost, C. (2002). The consequences of terrorism: Disentangling the effects of personal and national threat. Political Psychology, 23(3), 485-509. doi:10.11111/0162-895X.00295

Ho, M.-C., Shaw, D., Lin, S., \& Yao-Chu, C. (2008). How do disaster characteristics influence risk perception? Risk Analysis, 28(3), 635-643. doi:10.1111/j.15396924.2008.01040.x

Hollifield, M., Hewage, C., Gunawardena, C. N., Kodituwakku, P., \& Weerarathnege, K. (2008). Symptoms and coping in Sri Lanka 20-21 months after the 2004 Tsunami. The British Journal of Psychiatry, 192, 39-44.

doi:10.1192/bjp.bp.107.038422

Johannesson, K. B., Michel, P.-O., Hultman, C. M., Lindam, A., Arnberg, F., \& Lundin, T. (2009). Impact of exposure to trauma on posttraumatic stress disorder symptomatology in SwedishTourist Tsunami survivors. The Journal of Nervous and Mental Disease, 197(5), 316-323. doi:10.1097/NMD.0b013e3181a206f7

Kellens, W., Zaalberg, R., Vanneville, W., \& de Maeyer, P. (2011). An analysis of the public perception of flood risk on the Belgian coast. Risk Analysis, 31. doi:. doi:10.1111/j.1539-6924.2010.01571.x

Leach, J. (1994). Survival Psychology. Houndmills, Basingstoke, Hampshire and London: McMillian Press.

Leach, J. (2004). Why people 'freeze' in an emergency: Temporal and cognitive constraints on survival responses. Aviation, Space, and Environmental Medicine, 75(6), 539-542.

Marmar, C. R., Weiss, D. S., Schlenger, W. E., Fairbank, J. A., Jordan, B. K., Kulka, R. A., \& Hough, R. L. (1994). Peritraumatic dissociation and posttraumatic stress in male Vietnam theater veterans. The American Journal of Psychiatry, 151 (6), 902907.

Marmar, C. R., Weiss, D. S., \& Metzler, T. J. (1997). The Peritraumatic Dissociative Experiences Questionnaire. In: Wilson, J.P., Keane, T.M. (eds.) Assessing psychological trauma and posttraumatic stress disorder. New York: The Guilford Press.

Mellon, R. C., Papanikolau, V., \& Prodromitis, G. (2009). Locus of control and psychopathology in relation to levels of trauma and loss: Self-reports of 
Peloponnesian wildfire survivors. Journal of Traumatic Stress, 22(3), 189-196. doi:10.1002/jts.20411

Ozer, E. J., Best, S. R., Lipsey, T. L., \& Weiss, D. S. (2003). Predictors of posttraumatic stress disorder and symptoms in adults: A meta-analysis. Psychological Bulletin, 129(1), 52-73. doi:10.1037/0033-2909.129.1.52

Petersen, C., Schmidt, S., Power, M., \& Bullinger, M. (2005). Development and pilot testing of a health-related quality of life chronic generic module for children and adolescents with chronic health conditions: A European perspective. Quality of Life Research, 14, 1065-1077. doi:10.1007/s $11136-004-2575-z$

Prati, G., Catufi, V., \& Pietrantoni, L. (2012). The Umbria-Marche earthquake: Emotional and behavioral reactions to earth tremors. Disasters. Advance online publication. doi:10.1111/j.1467-7717.2011.01264.x

Preventionweb. (n.d.). Europe - Disaster statistics. Retrieved from http://www.preventionweb.net/english/countries/statistics/index_region.php?rid= 3.

Schlenger, W. E., Caddel, J. M., Ebert, L., Jordan, K., Rourke, K. M., Wilson, D., .... \& Kulka, R. A. (2002). Psychological reactions to terrorist attacks: Findings from the National Study of Americans' Reactions to September 11. Journal of the American Medical Association, 288(5), 581-588. doi:10.1001/jama.288.5.581

Shakespeare-Finch, J., \& Armstrong, J. (2010). Trauma type and type of posttrauma outcome differences between survivors of motor vehicle accidents, sexual assault, and bereavement. Journal of Loss and Trauma, 15, 69-82. doi:10.1080/15325020903373151

Shiloh, S., Guvenc, G., \& Onkal, D. (2007). Cognitive and emotional representations of terror attacks: A cross-cultural exploration. Risk Analysis, 27(2), 397-409. doi:10.1111/j.1539-6924.2007.00892.x

Silver, R. C., Holman, A., Mclntosh, D. N., Poulin, M., \& Gil-Rivas, V. (2002). Nationwide longitudinal study of psychological responses to September 11 . Journal of the American Medical Association, 288(10), 1235-1244. doi:10.1001/jama.288.10.1235 
Sotgiu, I., \& Galati, D. (2007). Long-term memory for traumatic events:

Experiences and emotional reactions during the 2000 flood in Italy. The Journal of Psychology, 141 (1), 91-108. doi:10.3200/JRLP.141.1.91-108

Steger, M. F., Frazier, P. A., \& Zacchanini, J. L. (2008). Terrorism in two cultures: Stress and growth following September 11 and the Madrid Bombings. Journal of Loss and Trauma, 13, 511-527. doi:10.1080/15325020802173660

Sumer, N., Karanci, A. N., Berument, S. K., \& Gunes, H. (2005). Personal resources, coping self-efficacy, and quake exposure as predictors of psychological distress following the 1999 earthquake in Turkey. Journal of Traumatic Stress, 18(4), $331-$ 342. doi:10.1002/jts.20032

Winkler, I., Matschinger, H., \& Angermayer, M. C. (2006). The WHOQOL-OLD. A Questionnaire for intercultural measuring of quality of life in the elderly. Psychotherapie, Psychosomatik, Medizinische Psychologie, 56, 63-69. doi:10.1055/s-2005-915334

\section{About the Authors}

Anna Grimm is a psychologist and PhD student at the Department of Health and Prevention at the University of Greifswald. Her main research interest is behavioural, emotional and cognitive responses of survivors of disasters. A second focus is on public risk perception of disasters.

Robert-Blum-Straße 13, 17487 Greifswald, Germany.

E-Mail: gesundheit.praevention@uni-greifswald.de

Dr. Lynn Hulse is a psychologist with the Fire Safety Engineering Group at the University of Greenwich. Her research interests include how civilians and emergency services personnel respond (i.e. their emotions, cognitions, and behaviours) to emergencies, and how this information may be of use to professionals involved in improving safety and the design of structures.

Prof. Dr. Silke Schmidt is a psychologist and head of the Department of Health and Prevention at the University of Greifswald. One of the areas of research is cross-cultural assessment of mental and physical health as well as quality of life. A second focus is on psychological preventions. 
Appendix A: One example of a scenario described at the beginning of the pilot form

\section{Simulation Scenario: Terror Attack}

Please imagine you are involved in the following situation: You are travelling in an underground train through London. It's approximately 8.30 a.m. and you are on your way to work. The train is just departing from the platform. All of a sudden you sense a big bang. First, you don' $t$ know what is going on, but you're immediately scared. You feel that you hurt your head during the blast. Your train is stopping in the middle of the tunnel. There's a power outage, so there's no light in your carriage anymore. People start getting nervous and panicking, and everybody tries to find out what is happening. There's no information given via loudspeakers, and no staff around to help people in the train. The doors are closed, so you and the other travellers are trapped. After approximately 10 minutes some people start breaking the door open and start evacuating from the carriage. You follow the other people through the tunnel back to the station. There's hardly any space between the train and the walls of the tunnel, so it's difficult for all of you to move forward. On your way out emergency services arrive and escort you out. The evacuation takes about 30 minutes. 